Published online 2017 April 13.

Abstract

\title{
Weight of Different Descriptors of Mammography
}

\author{
Masoumeh Gity ${ }^{1, *}$ \\ ${ }^{1}$ Associate Professor of Radiology, Tehran University of Medical Sciences, Tehran, Iran \\ "Corresponding author: Masoumeh Gity, MD, Associate Professor of Radiology, Tehran University of Medical Sciences, Tehran, Iran. E-mail: p_gity@yahoo.com
}

Received 2016 December 21; Accepted 2017 February 08.

\begin{abstract}
Mammography has been recognized as the best method of screening for breast cancer, for a long time. Although the sensitivity of mammography is acceptable, it has limited specificity and a radiologist should be aware of limitations and pitfalls. There are different positive findings in mammography which are descriptors of breast disease including benign lesions or breast cancer. In this talk I will explain different descriptors of mammography. These descriptors are different in weight toward predicting malignancy. For example, a mass is classified by the shape, margin and density. It has been discussed that some forms of "margin" have a high positive predictive value for malignancy. Also microcacifications as good descriptors for early malignant process are classified by their shape (morphology) and distribution. We will discuss the comparative weight of different descriptors as predictors of breast cancer.
\end{abstract}

This is an abstract presented in the 33rd Iranian congress of radiology (ICR) and the 15th congress of Iranian radiographic science association (IRSA). 